\title{
From Linear Logic to Cyclic Sharing
}

\author{
Masahito Hasegawa \\ Research Institute for Mathematical Sciences \\ Kyoto University \\ Kyoto, Japan \\ hassei@kurims.kyoto-u.ac.jp
}

\begin{abstract}
We present a translation from Multiplicative Exponential Linear Logic to a simply-typed lambda calculus with cyclic sharing. This translation is derived from a simple observation on the Intconstruction on traced monoidal categories. It turns out that the translation is a mixture of the call-by-name CPS translation and the Geometry of Interaction-based interpretation.
\end{abstract}

\section{Introduction}

It is often said that linear logic [7] is a resource-sensitive logic. Although this captures only one of the possible interpretations of linear logic, it is true that we can neatly represent various resource-conscious phenomena in linear logic. In particular, sharing of resources can be faithfully (and fully) interpreted in linear logic: there is a fully complete translation from the calculus of acyclic sharing graphs (term graphs) to the linear lambda calculus of the multiplicative exponential intuitionistic linear logic (MEILL) [2, 10], 1]

In this paper we consider a translation in the other direction, with suitably extended source and target calculi. Specifically, we give a translation from a linear lambda calculus for multiplicative exponential linear logic (MELL) to a simply-typed lambda calculus with cyclic sharing [9] (higher-order cyclic sharing theory [11]). The translation is derived from the following observation on traced monoidal categories and the Int-construction [15]:

Theorem 1 Consider a functor $F: \mathscr{C} \rightarrow \mathscr{D}$ from a category $\mathscr{C}$ to a traced symmetric monoidal category $\mathscr{D}$. Let $N: \mathscr{D} \rightarrow$ Int $\mathscr{D}$ be the canonical embedding of $\mathscr{D}$ to the compact closed category Int $\mathscr{D}$ obtained by the Int-construction. Then the composition $\mathscr{C} \stackrel{F}{\rightarrow} \mathscr{D} \stackrel{N}{\rightarrow}$ Int $\mathscr{D}$ has a right adjoint if and only if the functor $\mathscr{C} \stackrel{F}{\rightarrow} \mathscr{D} \stackrel{(-) \otimes D}{\longrightarrow} \mathscr{D}$ has a right adjoint for all $D$.

We give a proof of this theorem later, but it is embarrassingly short and easy; readers familiar with relevant concepts should have no difficulty in showing this by themselves.

Theorem 1, when combined with the categorical semantics, can be applied to turn a model of the lambda calculus with cyclic sharing to a model of MELL, hence to give a translation from MELL to the cyclic lambda calculus. Of course, the Int-construction has been widely used to construct models of linear logic, most notably in the context of Geometry of Interaction [8, 1]. Naturally our translation of the purely linear (multiplicative) part is essentially the same as the standard Int- or GoI-based interpretation. The novelty of this work lies in the treatment of the exponential modality !, which is a consequence of Theorem 1, One small surprise is that the interpretation of intuitionistic (or non-linear) implication

\footnotetext{
${ }^{1}$ In [2] the translation from Milner's action calculi [20] to MEILL is shown to be faithful (equationally complete), while action calculi correspond to calculi of acyclic sharing graphs [6 11]. Later the translation has been shown to be fully complete [10].
}

T. Ehrhard, M. Fernández, V. de Paiva, L. Tortora de Falco (Eds.):

Linearity-TLLA 2018

EPTCS 292, 2019, pp. 31-42 doi 10.4204/EPTCS.292.3 (c) M. Hasegawa

This work is licensed under the Creative Commons Attribution License. 
$\sigma \rightarrow \tau=! \sigma \multimap \tau$ agrees with the standard call-by-name continuation-passing style (CPS) translation. It turns out that this coincidence naturally follows from our categorical axiomatics.

We emphasize the semantics-directed nature of this work; the construction on semantic models comes first, which in turn gives rise to a syntactic translation whose soundness is guaranteed by construction.

Plan of this paper In Section 2, we recall the categorical structure relevant to this work and give a proof of Theorem 11. In Section 3, we recall the categorical semantics of MELL [25, 5, 14, 18] and the lambda calculus with cyclic sharing [11, 10, 22], and see how Theorem 1 gives rise to a translation from MELL to the cyclic lambda calculus. We then recall the calculi in Section 4 and describe the translation concretely (Section 5). In Section 6, we study the coincidence with the call-by-name CPS translation in terms of our model constructions.

\section{Traced monoidal categories and Int-construction}

\subsection{Preliminaries}

Recall that a trace [15] on a symmetric monoidal category $\mathscr{C}$ is a family of maps

$$
\operatorname{Tr}_{A, B}^{X}: \mathscr{C}(A \otimes X, B \otimes X) \rightarrow \mathscr{C}(A, B)
$$

subject to a few axioms. For $f: A \otimes X \rightarrow B \otimes X$, its trace $\operatorname{Tr}_{A, B}^{X} f: A \rightarrow B$ can be drawn as a "feedback":

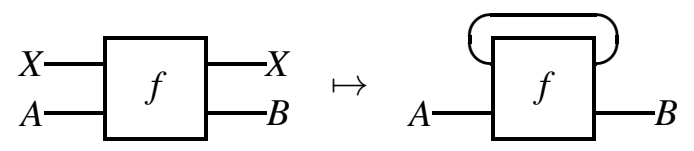

A traced symmetric monoidal category is a symmetric monoidal category equipped with a trace 2

A duality between two objects $A$ and $B$ of a monoidal category is determined by a pair of arrows $\eta: I \longrightarrow B \otimes A$ and $\varepsilon: A \otimes B \longrightarrow I$ such that the triangle equalities $\left(\varepsilon \otimes i d_{A}\right) \circ\left(i d_{A} \otimes \eta\right)=i d_{A}$ and $\left(i d_{B} \otimes \varepsilon\right) \circ\left(\eta \otimes i d_{B}\right)=i d_{B}$ hold. We say that $B$ is a right dual of $A$, and $A$ a left dual of $B$. A compact closed category is a symmetric monoidal category in which every object has a (right) dual.

Any monoidal full subcategory of a compact closed category is traced. Conversely, a traced symmetric monoidal category $\mathscr{C}$ gives rise to a compact closed category Int $\mathscr{C}$ to which $\mathscr{C}$ is fully faithfully embedded [15]. An object of Int $\mathscr{C}$ is a pair of objects of $\mathscr{C}$. A morphism $f:(X, U) \rightarrow(Y, V)$ in Int $\mathscr{C}$ is a morphism $f: X \otimes V \rightarrow Y \otimes U$ in $\mathscr{C}$. The composition of $f:(X, U) \rightarrow(Y, V)$ and $g:(Y, V) \rightarrow(Z, W)$ is

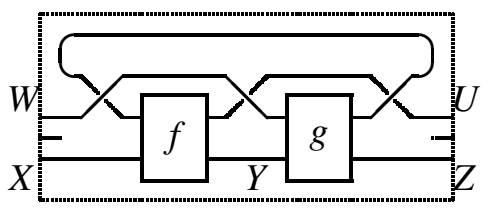

The tensor product of $\left(X_{1}, U_{1}\right)$ and $\left(X_{2}, U_{2}\right)$ is $\left(X_{1} \otimes X_{2}, U_{2} \otimes U_{1}\right)$, while the unit object is $(I, I)$. The dual of $(X, U)$ is $(U, X)$. There is a full faithful traced strong symmetric monoidal functor $N: \mathscr{C} \rightarrow \operatorname{Int} \mathscr{C}$ sending $X$ to $(X, I)$.

\footnotetext{
${ }^{2}$ The contents of this section are valid for traced balanced monoidal categories and tortile (ribbon) categories as well.
} 


\subsection{The embarrassingly easy theorem}

Now we are ready to show Theorem 1 .

Consider a functor $F: \mathscr{C} \rightarrow \mathscr{D}$ from a category $\mathscr{C}$ to a traced symmetric monoidal category $\mathscr{D}$. Then $N \circ F: \mathscr{C} \rightarrow \mathbf{I n t} \mathscr{D}$ has a right adjoint if and only if $F(-) \otimes D: \mathscr{C} \rightarrow \mathscr{D}$ has a right adjoint for all $D$.

The proof is immediate: we have

$$
\operatorname{Int} \mathscr{D}(N(F(C)),(Y, D))=\operatorname{Int} \mathscr{D}((F C, I),(Y, D)) \cong \mathscr{D}(F C \otimes D, Y)
$$

Obviously, $N \circ F$ has a right adjoint iff $F(-) \otimes D: \mathscr{C} \rightarrow \mathscr{D}$ has a right adjoint for every $D$.

Example 1 By letting $F$ be the identity functor on $\mathscr{D}$, Theorem $\square$ says that, for any traced symmetric monoidal category $\mathscr{D}, N: \mathscr{D} \rightarrow \mathbf{I n t} \mathscr{D}$ has a right adjoint if and only if $\mathscr{D}$ is closed [13].

Example 2 Let 1 be a one object one arrow category. Giving a functor from 1 to a traced symmetric monoidal category $\mathscr{D}$ is the same as giving an object $X$ of $\mathscr{D}$. That $\mathbf{1} \rightarrow \mathscr{D} \stackrel{N}{\rightarrow}$ Int $\mathscr{D}$ has a right adjoint means $N(X)=(X, I)$ is an initial object of Int $\mathscr{D}$. Therefore, Theorem 1 implies that, for an object $X$ of $\mathscr{D},(X, I)$ is an initial object of Int $\mathscr{D}$ if and only if $X \otimes D$ is an initial object of $\mathscr{D}$ for every $D$, i.e., $X$ is a distributive initial object in $\mathscr{D}$.

\section{Categorical models of linear logic and cyclic sharing}

\subsection{Categorical models of linear logic}

A categorical model of Multiplicative Exponential Linear Logic consists of a $*$-autonomous category $\mathscr{D}$ and a linear exponential comonad! on $\mathscr{D}$, where

- A $*$-autonomous category [4] is a symmetric monoidal closed category equipped with an object $\perp$ such that the canonical map $D \longrightarrow(D \multimap \perp) \multimap \perp$ is an isomorphism for every $D$.

- A linear exponential comonad [5, 14] on a symmetric monoidal category is a symmetric monoidal comonad such that its category of coalgebras is a category of commutative comonoids.

When ! is a linear exponential comonad, its category of coalgebras is a cartesian category (the induced monoidal product is cartesian). Conversely, any comonad induced by a symmetric monoidal adjunction between a cartesian category and a symmetric monoidal category is a linear exponential comonad [18].

\subsection{Categorical models of higher-order cyclic sharing}

A Freyd category [22] consists of a cartesian category $\mathscr{C}$, a symmetric (pre)monoidal category $\mathscr{D}$ and an identity-on-objects strict symmetric (pre)monoidal functor $F: \mathscr{C} \rightarrow \mathscr{D}$. Below we are interested in Freyd categories $F: \mathscr{C} \rightarrow \mathscr{D}$ in which $\mathscr{D}$ is monoidal (except Sec. 6 ). A Freyd category $F: \mathscr{C} \rightarrow \mathscr{D}$ is

- closed when the functor $F(-) \otimes D$ has a right adjoint (called Kleisli exponential) $D \Rightarrow(-)$ for each $D$.

- traced when $\mathscr{D}$ is traced.

We employ the approach taken in our previous work of modelling sharing graphs [11] using Freyd categories as the key semantic structure. 
1. For modelling first-order acyclic sharing, we use a Freyd category $F: \mathscr{C} \rightarrow \mathscr{D}$ where $\mathscr{D}$ is supposed to be monoidal. Values (including variables=wires) are interpreted in the cartesian category $\mathscr{C}$, while terms with sharing are interpreted in the monoidal category $\mathscr{D}$.

2. For modelling higher-order structure (i.e. lambda abstraction and application), we use a closed Freyd category. The adjunction $\mathscr{D}(F C \otimes D, E) \cong \mathscr{C}(C, D \Rightarrow E)$ says that currying turns a term to a value.

3. For modelling cyclic sharing, we use a traced Freyd category.

4. Finally, for modelling higher-order cyclic sharing (lambda calculus with cyclic sharing), we use a traced closed Freyd category.

\subsection{Relating models}

We apply Theorem 1 to turn a model of higher-order cyclic sharing (traced closed Freyd category) to a model of MELL ( $*$-autonomous category with a linear exponential comonad). As an immediate corollary to the theorem, we have:

Proposition 1 A traced Freyd category $F: \mathscr{C} \rightarrow \mathscr{D}$ is closed if and only if $N \circ F: \mathscr{C} \rightarrow$ Int $\mathscr{D}$ has a right adjoint.

Now suppose that we have a traced closed Freyd category $F: \mathscr{C} \rightarrow \mathscr{D}$ with $F(-) \otimes D \dashv D \Rightarrow(-)$ for each $D$. Then the strong symmetric monoidal functor $N F: \mathscr{C} \rightarrow \operatorname{Int} \mathscr{D}$ has a right adjoint sending $(X, U)$ to $U \Rightarrow X$. This symmetric monoidal adjunction gives rise to a linear exponential comonad ! on Int $\mathscr{D}$ sending $(X, U)$ to $(U \Rightarrow X, I)$. Since Int $\mathscr{D}$ is compact closed, it is $*$-autonomous. Thus Int $\mathscr{D}$ with! gives a model of MELL.

By applying this construction to the term model of the lambda calculus with cyclic sharing, we obtain a translation from MELL to the cyclic lambda calculus. This will be spelled out in the rest of this paper.

\section{The calculi}

\subsection{A lambda calculus with cyclic sharing}

We give a simply typed lambda calculus with cyclic sharing $\lambda_{\text {letrec }}$ (Figure1). This is essentially the same calculus as the higher-order cyclic sharing theory in [10], but slightly modified for a better match with semantic models (closed traced Freyd categories); the only differences are (i) treatment of product types (strictly associative or not) and (ii) treatment of variables (allowing variables on product types or not). Readers familar with Moggi's computational lambda calculus [21] should note that the $\lambda_{\text {letrec-calculus }}$ can be regarded as the commutative 3 version of the computational lambda calculus enriched with the recursive let-binding letrec for expressing cyclic sharing, as emphasized in [9].

As shown in [10], the $\lambda_{\text {letrec }}$-calculus is sound and complete for models given by closed traced Freyd categories.

\footnotetext{
${ }^{3}$ Here "commutativity" means the commutativity of effects let $x$ be $L$ in let $y$ be $M$ in $N=$ let $y$ be $M$ in let $x$ be $L$ in $N$. At the level of semantic models, this amounts to forcing the Freyd categories to be monoidal (rather than premonoidal), and to assuming the strong monads to be commutative.
} 
Types $\sigma::=b|\sigma \Rightarrow \sigma| \sigma \times \sigma \mid 1$

\section{Declarations, terms, values and contexts}

$$
\begin{array}{lll}
\text { Declarations } & D & :=\emptyset \mid x^{\sigma} \text { be } M, D \\
\text { Terms } & M & :=x\left|\lambda x^{\sigma} \cdot M\right| M M|(M, M)| \pi_{i} M|*| \text { letrec } D \text { in } M \\
\text { Values } & V & :=x\left|\lambda x^{\sigma} . M\right|(V, V)\left|\pi_{i} V\right| * \\
\text { Contexts } & C & :=[-]|C M| M C|(C, M)|(M, C)
\end{array}
$$

In the term letrec $D$ in $M$, the declaration $D$ must be non-empty.

Typing

$$
\begin{array}{ccc}
\frac{\Gamma, x: \sigma \vdash M: \tau}{\Gamma_{1}, x: \sigma, \Gamma_{2} \vdash x: \sigma} & \frac{\Gamma \vdash M: \sigma \Rightarrow \tau \quad \Gamma \vdash N: \sigma}{\Gamma \vdash \lambda x^{\sigma} . M: \sigma \Rightarrow \tau} & \frac{\Gamma \vdash M N: \tau}{\Gamma \vdash M: \sigma_{1} \times \sigma_{2}} \\
\frac{\Gamma \vdash M: \sigma \quad \Gamma \vdash N: \tau}{\Gamma \vdash(M, N): \sigma \times \tau} & \frac{\Gamma \vdash M \pi_{i}}{\Gamma \vdash \sigma_{i} M} & \overline{\Gamma \vdash *: 1} \\
\frac{\Gamma, x_{1}: \sigma_{1}, \ldots, x_{n}: \sigma_{n} \vdash M_{i}: \sigma_{i}(i=1, \ldots, n)}{\Gamma, x_{1}: \sigma_{1}, \ldots, x_{n}: \sigma_{n} \vdash N: \sigma} \\
\Gamma \vdash \text { letrec } x_{1}^{\sigma_{1}} \text { be } M_{1}, \ldots, x_{n}^{\sigma_{n}} \text { be } M_{n} \text { in } N: \sigma
\end{array}
$$

Notations We make use of the following syntax sugar.

$$
\begin{aligned}
\text { let } x^{\sigma} \text { be } M \text { in } N & \equiv\left(\lambda x^{\sigma} \cdot N\right) M \\
\lambda\left(x^{\sigma_{1}}, y^{\sigma_{2}}\right) \cdot M & \equiv \lambda z^{\sigma_{1} \times \sigma_{2}} \cdot M\left[x:=\pi_{1} z, y:=\pi_{2} z\right] \\
\text { let }\left(x^{\sigma_{1}}, y^{\sigma_{2}}\right) \text { be } M \text { in } N & \equiv\left(\lambda\left(x^{\sigma_{1}}, y^{\sigma_{2}}\right) \cdot N\right) M \\
\text { letrec } D_{1},(x, y) \text { be } M, D_{2} \text { in } N & \equiv \text { letrec } D_{1}, z \text { be } M, x \text { be } \pi_{1} z, y \text { be } \pi_{2} z, D_{2} \text { in } N
\end{aligned}
$$

\section{Axioms}

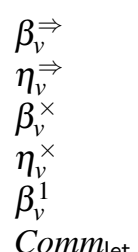

$\operatorname{Assoc}_{1}$

Assoc $_{2}$

Perm

Comm $_{\text {letrec }}$

$\sigma_{1}$

$\sigma_{2}$

$$
\begin{aligned}
(\lambda x . M) V & =M[x:=V] \\
\lambda x . V x & =V \quad(x \notin F V(V)) \\
\pi_{i}\left(V_{1}, V_{2}\right) & =V_{i} \\
\left(\pi_{1} V, \pi_{2} V\right) & =V \\
V & =* \quad(V: 1) \\
C[M] & =\text { let } x \text { be } M \text { in } C[x] \quad(x \notin F V(C))
\end{aligned}
$$

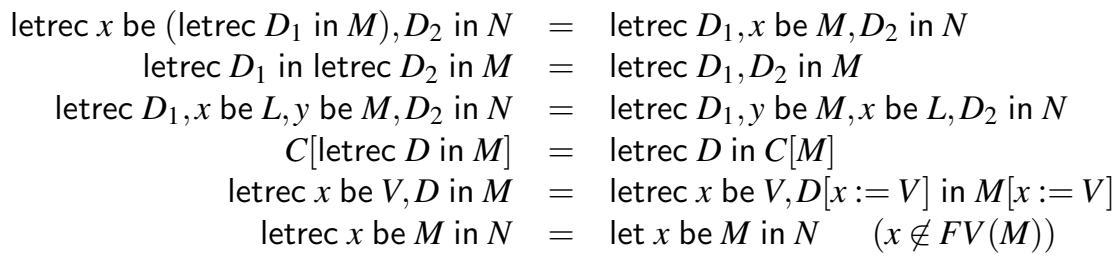

Figure 1: The $\lambda_{\text {letrec }}$-calculus 


\section{Types and terms}

$$
\begin{aligned}
& \sigma \quad::=b|\perp| \sigma \multimap \sigma \mid \sigma \rightarrow \sigma \\
& M::=x\left|\lambda x^{\sigma} . M\right| M M\left|\lambda x^{\sigma} . M\right| M @ M \mid \mathrm{C}_{\sigma} M
\end{aligned}
$$

where $b$ ranges over a set of base types. We may omit the type subscripts for ease of presentation.

Typing

$$
\begin{aligned}
& \overline{\Gamma_{1}, x: \sigma, \Gamma_{2} ; \emptyset \vdash x: \sigma} \text { Ax } \quad \overline{\Gamma ; x: \sigma \vdash x: \sigma}{ }_{\text {nonlinear }} x_{\text {linear }} \\
& \frac{\Gamma ; \Delta, x: \sigma \vdash M: \tau}{\Gamma ; \Delta \vdash \lambda x^{\sigma} . M: \sigma \multimap \tau} \multimap \text { Intr } \quad \frac{\Gamma ; \Delta_{1} \vdash M: \sigma \multimap \tau \quad \Gamma ; \Delta_{2} \vdash N: \sigma}{\Gamma ; \Delta_{1} \sharp \Delta_{2} \vdash M N: \tau} \multimap \text { Elim } \\
& \frac{\Gamma, x: \sigma ; \Delta \vdash M: \tau}{\Gamma ; \Delta \vdash \lambda x^{\sigma} . M: \sigma \rightarrow \tau} \rightarrow \text { Intr } \quad \frac{\Gamma ; \Delta \vdash M: \sigma \rightarrow \tau \quad \Gamma ; \emptyset \vdash N: \sigma}{\Gamma ; \Delta \vdash M @ N: \tau} \rightarrow \text { Elim } \\
& \frac{\Gamma ; \Delta \vdash M:(\sigma \multimap \perp) \multimap \perp}{\Gamma ; \Delta \vdash \mathrm{C}_{\sigma} M: \sigma} \text { Duality }
\end{aligned}
$$

where $\Delta_{1} \sharp \Delta_{2}$ is a merge of $\Delta_{1}$ and $\Delta_{2}$ [3]. When we introduce $\Delta_{1} \sharp \Delta_{2}$, it is assumed that there is no variable occurring both in $\Delta_{1}$ and $\Delta_{2}$.

Axioms

$$
\begin{array}{rrrlr}
\beta^{-} & (\lambda x . M) N & =M[x:=N] & \\
\eta^{\circ} & \lambda x \cdot M x & =M & \\
\beta^{\rightarrow} & (\lambda x \cdot M) @ N & =M[x:=N] & \\
\eta^{\rightarrow} & \lambda x . M @ x & =M & & (x \notin F V(M)) \\
\mathrm{C}_{1} & L\left(\mathrm{C}_{\sigma} M\right) & =M L & & (L: \sigma \multimap \perp) \\
\mathrm{C}_{2} & \mathrm{C}_{\sigma}(\lambda k . k M) & =M &
\end{array}
$$

Figure 2: Dual Classical Linear Logic (DCLL)

\subsection{A linear lambda calculus for MELL}

As the calculus for MELL, we use DCLL (dual classical linear logic) [12], as recalled in Figure 2. DCLL is an extension of DILL (dual intuitionistic linear logic) of Barber and Plotkin [3], but has just linear implication $\multimap$, non-linear implication $\rightarrow$ and the falsity type $\perp$ as the primitive type constructs. Terms are built from variables, the linear lambda abstraction $\lambda x^{\sigma} . M$ and application $M N$, non-linear lambda abstraction $\lambda x^{\sigma} . M$ and application $M @ N$, and the double-negation elimination $C_{\sigma} M$. Like DILL, DCLL employs a dual-context formulation, where a typing judgement takes the form $\Gamma ; \Delta \vdash M: \tau$ in which $\Gamma$ represents a non-linear (intuitionistic) context whose variables can be used as many times as we like whereas $\Delta$ is a linear context whose variables are used exactly once. The equational theory has just the $\beta \eta$-axioms together with two axioms for the isomorphism $(\sigma \multimap \perp) \multimap \perp \cong \sigma$.

Despite its simplicity, DCLL is sound and complete for models given by $*$-autonomous categories with a linear exponential comonad, and can express other connectives and proofs of MELL [12], e.g., $I=\perp \multimap \perp, \sigma \otimes \tau=(\sigma \multimap \tau \multimap \perp) \multimap \perp$ and $! \sigma=(\sigma \rightarrow \perp) \multimap \perp$. 


\section{The translation}

\subsection{From DCLL to the $\lambda_{\text {letrec }}$-calculus}

We spell out the translation from DCLL to the $\lambda_{\text {letrec }}$-calculus derived from the semantic construction in Section 3, For each base type $b$ of DCLL, fix types $b^{+}$and $b^{-}$of the $\lambda_{\text {letrec }}$-calculus. For a typing judgement

$$
\Gamma ; \Delta \vdash M: \sigma
$$

in DCLL, its translation in the $\lambda_{\text {letrec }}$-calculus

$$
\Gamma^{-\Rightarrow+}, \Delta^{+} \vdash[[M]]^{\Delta}: \sigma^{-} \Rightarrow\left(\sigma^{+} \times \Delta^{-}\right)
$$

is given as follows, where

$$
\begin{aligned}
\left(x_{1}: \sigma_{1}, \ldots, x_{m}: \sigma_{m}\right)^{-\Rightarrow+} & =x_{1}: \sigma_{1}^{+} \Rightarrow \sigma_{1}^{-}, \ldots, x_{m}: \sigma_{m}^{+} \Rightarrow \sigma_{m}^{-} \\
\left(y_{1}: \tau_{1}, \ldots, y_{n}: \tau_{n}\right)^{+} & =y_{1}: \tau_{1}^{+}, \ldots, y_{n}: \tau_{n}^{+} \\
\left(y_{1}: \tau_{1}, \ldots, y_{n}: \tau_{n}\right)^{-} & =\tau_{1}^{-} \times \cdots \times \tau_{n}^{-}
\end{aligned}
$$

and the translation of types and terms are inductively given as follows. Figure 3 gives a summary of the translation.

Remark 1 In describing the translation, we pretend as if the product types in the $\lambda_{\text {letrec-calculus are }}$ strictly associative, e.g., we identify $((x, y), z):\left(\sigma_{1} \times \sigma_{2}\right) \times \sigma_{3}$ with $(x,(y, z)): \sigma_{1} \times\left(\sigma_{2} \times \sigma_{3}\right)$ and $(x, *)$ : $\sigma \times 1$ with $x: \sigma$. This makes the description of translation much simpler. Alternatively, we could make use of the original higher-order cyclic sharing theory [11] whose products are strictly associative.

\section{Translation of types}

$$
\begin{array}{rlrlrl}
(\sigma \rightarrow \tau)^{+} & =\tau^{+} & (\sigma \rightarrow \tau)^{-} & =\left(\sigma^{-} \Rightarrow \sigma^{+}\right) \times \tau^{-} \\
(\sigma \multimap \tau)^{+} & =\tau^{+} \times \sigma^{-} & (\sigma \multimap \tau)^{-} & =\sigma^{+} \times \tau^{-} \\
\perp^{+} & =1 & & \perp^{-} & =1
\end{array}
$$

\section{Translation of terms}

$$
\begin{aligned}
{[[x]]^{\emptyset} } & =\lambda k \cdot x k \\
{[[y]]^{y: \sigma} } & =\lambda k \cdot(y, k) \\
{\left[[\lambda x \cdot M]^{\Delta}\right.} & =\lambda(x, k) \cdot\left[[M]^{\Delta} k\right. \\
{\left[[M @ N]^{\Delta}\right.} & =\lambda k \cdot\left[[ M ] ^ { \Delta } \left(\left[[N]^{\emptyset}, k\right)\right.\right. \\
{\left[\left[\lambda y^{\sigma} \cdot M\right]^{\Delta}\right.} & =\lambda(y, k) \cdot[[M]]^{\Delta, y: \sigma} k \\
{[[M N]]^{\Delta_{1} \sharp \Delta_{2}} } & =\lambda k \cdot\left(\text { letrec }\left(u, \overrightarrow{z_{2}}\right) \text { be }\left[[N]^{\Delta_{2}} h,\left(v, h, \vec{z}_{1}\right) \text { be }[[M]]^{\Delta_{1}}(u, k) \text { in }\left(v, \overrightarrow{z_{1}} \sharp \overrightarrow{z_{2}}\right)\right)\right. \\
{[[C M]]^{\Delta} } & =\left[[M]^{\Delta}\right.
\end{aligned}
$$

Note that the translation of linear constructs agrees with the standard Int- or GoI-based interpretation. For instance, the linear application $[[M N]]^{\Delta_{1}, \Delta_{2}}$ is graphically presented as follows - it is an instance of the composition in compact closed categories obtained by the Int-construction.

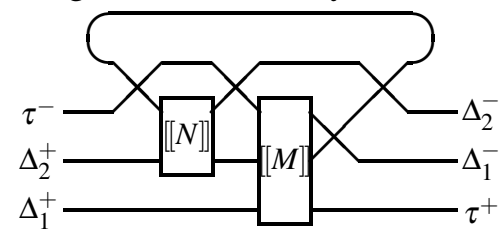




\begin{tabular}{|c|c|}
\hline$\Gamma ; \Delta \vdash_{\mathbf{D C L L}} M: \sigma$ & $\Gamma^{-\Rightarrow+}, \Delta^{+} \vdash_{\lambda_{\text {letrec }}} \llbracket\left[M \rrbracket: \sigma^{-} \Rightarrow\left(\sigma^{+} \times \Delta^{-}\right)\right.$ \\
\hline$\overline{\Gamma_{1}, x: \sigma, \Gamma_{2} ; \emptyset \vdash x: \sigma} A x_{\text {non linear }}$ & $\overline{\Gamma_{1}^{-\Rightarrow+}, x: \sigma^{-} \Rightarrow \sigma^{+}, \Gamma_{2}^{-\Rightarrow}{ }^{+} \vdash \lambda k^{\sigma^{-}} . x k: \sigma^{-} \Rightarrow \sigma^{+}}$ \\
\hline$\overline{\Gamma ; x: \sigma \vdash x: \sigma} A x_{\text {linear }}$ & $\overline{\Gamma^{-\Rightarrow+}, x: \sigma^{+} \vdash \lambda k^{\sigma^{-}} .(x, k): \sigma^{-} \Rightarrow\left(\sigma^{+} \times \sigma^{-}\right)}$ \\
\hline$\frac{\Gamma ; \Delta, x: \sigma \vdash M: \tau}{\Gamma ; \Delta \vdash \lambda x^{\sigma} . M: \sigma \multimap \tau} \multimap$ Intr & $\frac{\Gamma^{-\Rightarrow+}, \Delta^{+}, x: \sigma^{+} \vdash[[M]]^{\Delta, x: \sigma}: \tau^{-} \Rightarrow\left(\tau^{+} \times \Delta^{-}\right)}{\Gamma^{-\Rightarrow}, \Delta^{+} \vdash \lambda(x, k) \cdot\left[[M]^{\Delta, x: \sigma} k:\left(\sigma^{+} \times \tau^{-}\right) \Rightarrow\left(\tau^{+} \times \Delta^{-}\right)\right.}$ \\
\hline$\frac{\Gamma ; \Delta_{1} \vdash M: \sigma \multimap \tau \quad \Gamma ; \Delta_{2} \vdash N: \sigma}{\Gamma ; \Delta_{1} \sharp \Delta_{2} \vdash M N: \tau} \multimap$ Elim & $\begin{array}{c}\Gamma^{-\Rightarrow+}, \Delta_{1}^{+} \vdash \llbracket[M]^{\Delta_{1}}:\left(\sigma^{+} \times \tau^{-}\right) \Rightarrow\left(\tau^{+} \times \sigma^{-} \times \Delta_{1}^{-}\right) \\
\Gamma^{-\Rightarrow+}, \Delta_{2}^{+} \vdash[\| N]^{\Delta_{2}}: \sigma^{-} \Rightarrow\left(\sigma^{+} \times \Delta_{2}^{-}\right) \\
\Gamma^{-\Rightarrow+},\left(\Delta_{1} \sharp \Delta_{2}\right)^{+} \vdash \\
\left.\lambda k^{\tau^{-}} . \text {letrec }\left(u: \sigma^{+}, \overrightarrow{z_{2}}: \Delta_{2}^{-}\right) \text {be } \llbracket[N]\right]^{\Delta_{2}} h, \\
\left(v: \tau^{+}, h: \sigma^{-}, \overrightarrow{z_{1}}: \Delta_{1}^{-}\right) \text {be }\left[[M]^{\Delta_{1}}(u, k)\right. \\
\text { in }\left(v, \overrightarrow{z_{1}} \sharp \overrightarrow{z_{2}}\right): \tau^{-} \Rightarrow\left(\tau^{+} \times\left(\Delta_{1} \sharp \Delta_{2}\right)^{-}\right)\end{array}$ \\
\hline$\frac{\Gamma, x: \sigma ; \Delta \vdash M: \tau}{\Gamma ; \Delta \vdash \lambda x^{\sigma} . M: \sigma \rightarrow \tau} \rightarrow$ Intr & $\frac{\Gamma^{-\Rightarrow+}, x: \sigma^{-} \Rightarrow \sigma^{+}, \Delta^{+} \vdash[\llbracket M]^{\Delta}: \tau^{-} \Rightarrow\left(\tau^{+} \times \Delta^{-}\right)}{\Gamma^{-\Rightarrow+}, \Delta^{+} \vdash \lambda(x, k) \cdot[M]^{\Delta} k:\left(\left(\sigma^{-} \Rightarrow \sigma^{+}\right) \times \tau^{-}\right) \Rightarrow\left(\tau^{+} \times \Delta^{-}\right)}$ \\
\hline$\frac{\Gamma ; \Delta \vdash M: \sigma \rightarrow \tau \quad \Gamma ; \emptyset \vdash N: \sigma}{\Gamma ; \Delta \vdash M @ N: \tau} \rightarrow$ Elim & $\begin{array}{c}\Gamma^{-\Rightarrow+}, \Delta^{+} \vdash[[M]]^{\Delta}:\left(\left(\sigma^{-} \Rightarrow \sigma^{+}\right) \times \tau^{-}\right) \Rightarrow\left(\tau^{+} \times \Delta^{-}\right) \\
\Gamma^{-\Rightarrow+} \vdash\left[[N]^{\emptyset}: \sigma^{-} \Rightarrow \sigma^{+}\right. \\
\Gamma^{-\Rightarrow+}, \Delta^{+} \vdash \lambda k \cdot\left[[M]^{\Delta}\left(\left[[N]^{\emptyset}, k\right): \tau^{-} \Rightarrow\left(\tau^{+} \times \Delta^{-}\right)\right.\right.\end{array}$ \\
\hline$\frac{\Gamma ; \Delta \vdash M:(\sigma \multimap \perp) \multimap \perp}{\Gamma ; \Delta \vdash C_{\sigma} M: \sigma}$ Duality & $\frac{\Gamma^{-\Rightarrow+}, \Delta^{+} \vdash\left[[M]^{\Delta}: \sigma^{-} \Rightarrow\left(\sigma^{+} \times \Delta^{-}\right)\right.}{\Gamma^{-\Rightarrow+}, \Delta^{+} \vdash\left[[M]^{\Delta}: \sigma^{-} \Rightarrow\left(\sigma^{+} \times \Delta^{-}\right)\right.}$ \\
\hline
\end{tabular}

Figure 3: Summary of the translation

The soundness of the translation follows by definition:

Proposition 2 (type soundness) If the typing judgement $\Gamma ; \Delta \vdash M: \sigma$ is derivable in DCLL, then $\Gamma^{-\Rightarrow+}, \Delta^{+} \vdash[[M]]^{\Delta}: \sigma^{-} \Rightarrow\left(\sigma^{+} \times \Delta^{-}\right)$is derivable in the $\lambda_{\text {letrec }}$ calculus.

Proposition 3 (equational soundness) If the equation $\Gamma ; \Delta \vdash M=N: \sigma$ is derivable in DCLL, then $\Gamma^{-\Rightarrow+}, \Delta^{+} \vdash[[M]]=[[N]]$ is derivable in the $\lambda_{\text {letrec }}$-calculus.

We shall note that our translation is not equationally complete, because of the coherence of compact closed categories. For instance, the two proofs of $((\sigma \multimap I) \multimap I) \multimap I \vdash((\sigma \multimap I) \multimap I) \multimap I$ (where $I=\perp \multimap \perp$ ) get the same interpretation (the triple-unit problem [16]).

\subsection{Examples}

One might expect that the translation of a well-typed term of DCLL would be equal to a letrec-free term in the $\lambda_{\text {letrec }}$-calculus. The following example shows that it is not the case; we cannot eliminate letrec even when we restrict our attention to terms in $\beta$-normal form. Consider the term

$$
f: \sigma \multimap \tau, g: \tau \multimap \delta ; x: \sigma \vdash_{\text {DCLL }} g(f x): \delta
$$


with non-linear variables $f, g$ and a linear variable $x$. This term is interpreted as

$$
\begin{aligned}
& f:\left(\sigma^{+} \times \tau^{-}\right) \Rightarrow\left(\tau^{+} \times \sigma^{-}\right), \\
& g:\left(\tau^{+} \times \delta^{-}\right) \Rightarrow\left(\delta^{+} \times \tau^{-}\right), \quad \vdash \lambda k^{\delta^{-}} .\left(\text {letrec } \begin{array}{c}
\left(u^{\tau^{+}}, z^{\sigma^{-}}\right) \text {be } f(x, h), \\
\left(v^{\delta^{+}}, h^{\tau^{-}}\right) \text {be } g(u, k)
\end{array} \text { in }(v, z)\right): \delta^{-} \Rightarrow\left(\delta^{+} \times \sigma^{-}\right)
\end{aligned}
$$

This is precisely the composition in the Int-category.

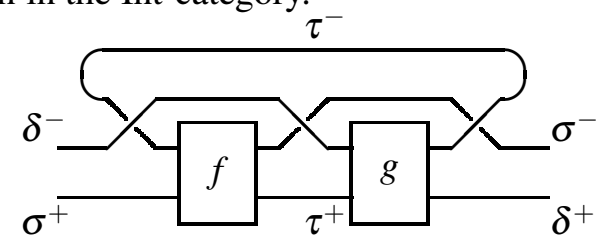

Note that the use of non-linear variables is essential. For instance, the interpretation of the following term

$$
\emptyset ; f: \sigma \multimap \tau, g: \tau \multimap \delta, x: \sigma \vdash_{\text {DCLL }} g(f x): \delta
$$

with linear variables $f, g, x$ is equal to the letrec-free term

$f: \tau^{+} \times \sigma^{-}, g: \delta^{+} \times \tau^{-}, x: \sigma^{+} \vdash \lambda k^{\delta^{-}} .\left(\pi_{1} g, x, \pi_{2} g, \pi_{1} f, k, \pi_{2} f\right): \delta^{-} \Rightarrow\left(\delta^{+} \times \sigma^{+} \times \tau^{-} \times \tau^{+} \times \delta^{-} \times \sigma^{-}\right)$

since the linear variables are simply interpreted as wirings.

\section{Relation to the call-by-name CPS translation}

The translation of non-linear variables, non-linear lambda abstraction and non-linear application agrees with the (Streicher-style) call-by-name CPS translation [26]

$$
[[x]]^{\emptyset}=\lambda k \cdot x k \quad[[\lambda x \cdot M]]^{\emptyset}=\lambda(x, k) \cdot[[M]]^{\emptyset} k \quad[[M @ N]]^{\emptyset}=\lambda k \cdot[[M]]^{\emptyset}\left([[N]]^{\emptyset}, k\right)
$$

though our translation does not assume a single fixed answer type. This is because the translation of non-linear types picks up a cartesian closed category derived from the closed Freyd category:

Proposition 4 (folklore?) Suppose that $F: \mathscr{C} \rightarrow \mathscr{D}$ is a closed Freyd category (where $\mathscr{D}$ can be premonoidal). Then there is a cartesian closed full subcategory of $\mathscr{C}$ whose objects are finite products of objects of the form $D \Rightarrow E$.

$$
\mathscr{C}\left(\Gamma \times(D \Rightarrow E), D^{\prime} \Rightarrow E^{\prime}\right) \cong \mathscr{C}\left(\Gamma,\left((D \Rightarrow E) \times D^{\prime}\right) \Rightarrow E^{\prime}\right)
$$

In the case of traced closed Freyd categories, this cartesian closed category is where the interpretation of non-linear abstraction and application takes place:

$$
\text { Int } \mathscr{D}\left(!\left(X_{1}, U_{1}\right) \otimes \cdots \otimes !\left(X_{n}, U_{n}\right),(Y, V)\right) \cong \mathscr{C}\left(\left(U_{1} \Rightarrow X_{1}\right) \times \cdots \times\left(U_{n} \Rightarrow X_{n}\right), V \Rightarrow Y\right)
$$

By letting the codomains $E$ and $E^{\prime}$ (or $X_{i}$ and $Y$ ) be a fixed answer type, we obtain the standard CPS semantics.

This situation can be summarized as the following picture. The outer square expresses our model constructions from traced closed Freyd categories, while the inner triangle shows the induced syntactic translations. Commutativity at the level of semantic model consructions guarantees commutativity of syntactic translations. (Since we employ DCLL as the language for MELL, the Girard translation from the simply typed lambda calculus to MELL is just an inclusion in our formulation.) 


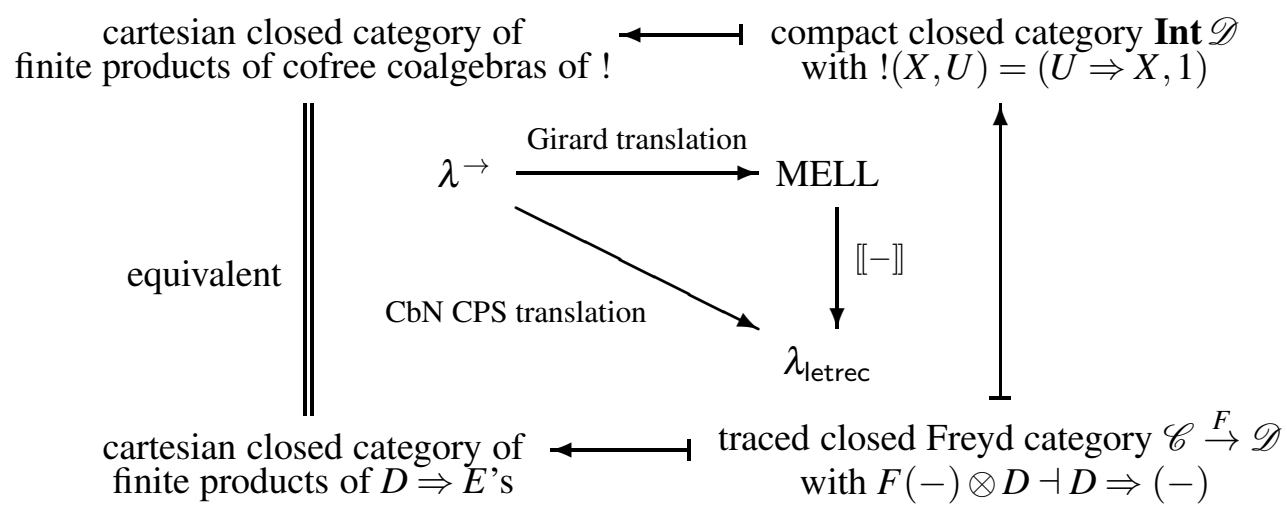

\section{Concluding remarks}

We gave a translation from MELL to a lambda calculus with cyclic sharing. This translation is derived from an easy theorem (Theorem 1) on traced monoidal categories. Once we know the theorem, it is fairly routine to derive the syntactic translation. Perhaps the most difficult part would be to establish the appropriate syntax and categorical semantics of MELL and cyclic sharing, which had been sorted out many yeas ago. We do not claim that this translation would immediately lead to a practical application, but hope that it makes an interesting case of the semantics-driven approach to program transformations.

Our translation can be seen as a combination of GoI interpretation and CPS translation. Although we could have used any calculi/proof nets which are sound for $*$-autonomous categories with a linear exponential comonad, the simple design of DCLL allows us to simplify the description of the translation a lot. In particular, in this formulation with linear/non-linear implications, the relation to the CPS translation is very easily observed. It seems that this relation to CPS semantics is new; Schöpp [24] observed coincidence of CPS semantics and Int-interpretation in a different setting, but we are yet to see if there is any formal relationship between his work and ours. It would also be meaningful to investigate the connection between our translation and game semantics (or tensorial logic) along the work of Melliès and Tabareau [19], where they study categorical structures closely related to ours.

Finally we shall mention a relation to the categorical semantics (and game semantics) of the $\pi$ calculus in [17, 23]. The models in these work form traced closed Freyd categories, thus are instances of the structure considered in this paper. It would be interesting to combine our translation and their work, which might lead to new relation between linear logic and concurrency theory.

Acknowledgements I thank Ken Sakayori and Takeshi Tsukada for stimulating discussions related to this work. This work was supported by JSPS KAKENHI Grant Numbers JP15K00013, JP18K11165 and JST ERATO Grant Number JPMJER1603, Japan.

\section{References}

[1] S. Abramsky, E. Haghverdi \& P.J. Scott (2002): Geometry of interaction and linear combinatory algebras. Mathematical Structures in Computer Science 12(5), pp. 625-665, doi:10.1017/S0960129502003730.

[2] A. Barber, P. Gardner, M. Hasegawa \& G. Plotkin (1998): From action calculi to linear logic. In: Computer Science Logic (CSL'97), Selected Papers, Lecture Notes in Computer Science 1414, Springer-Verlag, pp. 78-97, doi:10.1007/BFb0028008. 
[3] A. Barber \& G.D. Plotkin (1997): Dual intuitionistic linear logic. Unpublished draft. An early version appeared as a technical report ECS-LFCS-96-347, LFCS, University of Edinburgh.

[4] M. Barr (1979): *-Autonomous Categories. Lecture Notes in Mathematics 752, Springer-Verlag, doi:10. $1007 / \mathrm{BFb} 0064582$.

[5] G.M. Bierman (1995): What is a categorical model of intuitionistic linear logic? In: Proceedings of TLCA'95, Lecture Notes in Computer Science 902, Springer-Verlag, pp. 78-93, doi:10.1007/BFb0014046.

[6] P. Gardner \& M. Hasegawa (1997): Types and models for higher-order action calculi. In: Proceedings of TACS'97, Lecture Notes in Computer Science 1281, Springer-Verlag, pp. 583-603, doi:10.1007/ BFb0014569.

[7] J.-Y. Girard (1987): Linear logic. Theoretical Computer Science 50, pp. 1-102, doi:10.1016/ 0304-3975 (87) 90045-4.

[8] J.-Y. Girard (1989): Towards a geometry of interaction. In: Categories in Computer Science and Logic, Contemporary Mathematics 92, AMS, pp. 69-108, doi:10.1090/conm/092/1003197.

[9] M. Hasegawa (1997): Recursion from cyclic sharing: traced monoidal categories and models of cyclic lambda calculi. In: Proceedings of TLCA'97, Lecture Notes in Computer Science 1210, Springer-Verlag, pp. 196-213, doi:10.1007/3-540-62688-3_37.

[10] M. Hasegawa (1999): Categorical glueing and logical predicates for models of linear logic. Available at http://www.kurims.kyoto-u.ac.jp/ hassei/papers/full.pdf. Preprint RIMS-1223.

[11] M. Hasegawa (1999): Models of Sharing Graphs: A Categorical Semantics of let and letrec. Distingushed Dissertations Series, Springer-Verlag, doi:10.1007/978-1-4471-0865-8. Also available as PhD thesis ECS-LFCS-97-360, LFCS, University of Edinburgh.

[12] M. Hasegawa (2005): Classical linear logic of implications. Mathematical Structures in Computer Science 15(2), pp. 323-342, doi:10.1017/S0960129504004621.

[13] M. Hasegawa (2009): On traced monoidal closed categories. Mathematical Structures in Computer Science 19(2), pp. 217-244, doi:10.1017/S0960129508007184.

[14] J.M.E. Hyland \& A. Schalk (2003): Glueing and orthogonality for models of linear logic. Theoretical Computer Science 294(1-2), pp. 183-231, doi:10.1016/S0304-3975(01)00241-9.

[15] A. Joyal, R. Street \& D. Verity (1996): Traced monoidal categories. Mathematical Proceedings of the Cambridge Philosophical Society 119(3), pp. 447-468, doi:10.1017/S0305004100074338.

[16] G.M. Kelly \& S. Mac Lane (1971): Coherence in closed categories. Journal of Pure and Applied Algebra 1(1), pp. 97-140, doi:10.1016/0022-4049(71)90013-2.

[17] J. Laird (2005): A game semantics of the asynchronous $\pi$-calculus. In: Proceedings of CONCUR 2005, Lecture Notes in Computer Science 3653, Springer, pp. 51-65, doi:10.1007/11539452_8.

[18] P.-A. Melliès (2009): Categorical semantics of linear logic. In: Interactive Models of Computation and Program Behavior, Panoramas et Synthèses 27, Société Mathématique de France, pp. 1-196.

[19] P.-A. Melliès \& N. Tabareau (2010): Resource modalities in tensor logic. Annals in Pure and Applied Logic 161(5), pp. 632-653, doi:10.1016/j.apal.2009.07.018.

[20] R. Milner (1996): Calculi for interaction. Acta Inf. 33(8), pp. 707-737, doi:10.1007/BF03036472.

[21] E. Moggi (1989): Computational lambda-calculus and monads. In: Proceedings of LICS'89, IEEE Computer Society, pp. 14-23, doi:10.1109/LICS.1989.39155.

[22] J. Power \& H. Thielecke (1999): Closed Freyd-and kappa-categories. In: Proceedings of ICALP'99, Lecture Notes in Computer Science 1644, Springer-Verlag, pp. 625-634, doi:10.1007/3-540-48523-6_59.

[23] K. Sakayori \& T.Tsukada (2017): A truly concurrent game model of the asynchronous $\pi$-calculus. In: Proceedings of FOSSACS 2017, Lecture Notes in Computer Science 10203, Springer-Verlag, pp. 389-406, doi:10.1007/978-3-662-54458-7_23. 
[24] U. Schöpp (2014): On the relation of interaction semantics to continuations and defunctionalization. Logical Methods in Computer Science 10(4), doi:10.2168/LMCS-10 (4:10) 2014.

[25] R.A.G. Seely (1989): Linear logic, *-autonomous categories and cofree coalgebras. In: Categories in Computer Science and Logic, Contemporary Mathematics 92, AMS, pp. 371-389, doi:10.1090/conm/092/ 1003210 .

[26] T. Streicher \& B. Reus (1998): Classical logic, continuation semantics and abstract machines. Journal of Functional Programming 8(6), pp. 543-572, doi:10.1017/S0956796898003141. 